\title{
VIAJAR CON LA TRADICIÓN: LOS PESCADORES ARTESANALES ANTE LA PESCA-TURISMO EN LA COMUNIDAD VALENCIANA (ESPAÑA) ${ }^{1}$
}

\author{
Paloma Herrera-Racionero* \\ Luis Miret-Pastor* \\ Universidad Politécnica de Valencia \\ Emmánuel Lizcano** \\ Universidad Nacional de Educación a Distancia
}

\section{RESUMEN}

A pesar del potencial de los puertos españoles, la pesca-turismo no acaba de implantarse en nuestro país. Solucionados, en principio, los problemas legislativos, la actitud de los pescadores frente a esta actividad parece uno de los asuntos clave que determinará su éxito o fracaso. Este trabajo se centra en analizar la percepción que los pescadores tienen de la pesca-turismo, concretamente, en las oportunidades y amenazas que perciben. Para ello se ha llevado a cabo un estudio cualitativo basado en el análisis en profundidad de 32 entrevistas a pescadores de la Comunidad Valenciana (España).

Palabras clave: Pesca-turismo, sostenibilidad socio-ambiental, pesca artesanal, burocracia, análisis cualitativo.

Recibido: 20 de julio de 2016

Devuelto para su revisión: 23 de noviembre de 2016

Aceptado: 29 de enero de 2017

* Escuela Politécnica Superior de Gandía. Universidad Politécnica de Valencia. C/Paranimf, nº 1.46730 Grau de Gandía VALENCIA (España).E-mail: paherra@esp.upv.es, luimipas@esp.ipv.es

** Departamento de Sociología I. Facultad de Ciencias Políticas y Sociología. Universidad Nacional de Educación a Distancia. C/ Obispo Trejo, 2. 28040 MADRID (España).E-mail: elizcano@poli.uned.es

1 Este trabajo forma parte del proyecto de investigación titulado "Las posibilidades del turismo pesquero en la Comunidad Valenciana" (ref. GV/2015/070) financiado por la Generalitat Valenciana. 


\title{
Traveling with tradition: artisanal fishermen and fishing tourism in Valencian Region (Spain)
}

\begin{abstract}
Despite the potential of Spanish ports, fishing tourism has not finished implanted in our country. Once the legislative problems were solved, the attitude of fishermen of this activity seems one of the key issues that will determine its success or failure. This paper focuses on analysing the fishermen' perception about fishing-tourism, specifically on the opportunities and threats they perceive. We have carried out a qualitative study based on in-depth analysis of 32 interviews with fishermen in the Valencia region (Spain)
\end{abstract}

Keywords: Fishing tourism, social and environmental sustainability, artisanal fishery, bureaucracy, qualitative analysis.

\section{INTRODUCCIÓN}

La crisis socio-ambiental que está viviendo la pesca tradicional en el Mediterráneo, con importantes costes económicos y culturales, está estimulando la búsqueda de alternativas que concilien la supervivencia de la pesca artesanal, con gran arraigo en las poblaciones litorales, con la necesaria sostenibilidad ambiental. Desde un enfoque ecosistémico para la gestión de recursos naturales, se están promoviendo diferentes iniciativas que, como el "environmentally-oriented tourism" (Fairweather et al., 2005; Job y Paesler, 2013) o "cooperative tourism" (Farrel, 2008) logren esa conciliación.

En este contexto, el turismo pesquero está llamado a adquirir una importancia creciente. Por una parte, encontramos un sector pesquero tradicional, sumido en una profunda crisis social, ambiental y económica; por otra parte, compartiendo territorio y recursos, encontramos un sector turístico líder a nivel mundial pero que busca renovarse para huir de un modelo muy maduro y estandarizado de sol y playa.

Las posibles sinergias entre turismo y pesca no han pasado desapercibidas ni para los pescadores, ni para la administración, ni para el sector turístico. La Unión Europea promueve una visión ecosistémica para su Política Integrada Marítima (IPM) y señala la pesca-turismo como uno de los ejes para lograr una pesca más sostenible (Comisión Europea, 2007). Tales sinergias suponen también una aplicación concreta dentro de la estrategia comunitaria de Gestión Integrada de Zonas Costeras (GIZC). Sin embargo, incluso entre los países europeos con mayor potencial turístico, el desarrollo de la pesca-turismo aún está en una fase embrionaria y, en todo caso, es muy irregular. Países como Italia o Francia fueron pioneros en su implantación mientras que otros países, como España, tenían explícitamente prohibido embarcar turistas hasta no hace mucho tiempo.

Muchas actividades de turismo pesquero representan una propuesta novedosa que responde a la necesaria diversificación tanto de la actividad pesquera como de la turística. Por un lado, respecto al turismo, es indudable que se encuentra en una época de cambios estructurales (Onder et al., 2009; Papatheodorou et al., 2010), los cuales son particularmente importantes en el modelo turístico tradicional. De acuerdo con la teoría del ciclo 
de vida de los destinos turísticos (Butler, 1980), buena parte del turismo en nuestra costa mediterránea estaría formado por destinos maduros, paradigmáticos de lo que se conoce como turismo de segunda generación, según la clasificación de Knowles y Curtis (1999). Este turismo suele identificarse con modelos insostenibles y en declive (Agarwal, 2002), sin embargo, en los últimos años, como consecuencia de la aparición de nuevas tecnologías y estilos de vida, ha aparecido un nuevo modelo turístico llamado de tercera generación o neo-fordista (Ioannides y Debbagge, 1998; Torres, 2002), caracterizado por un mayor control de la planificación por parte del cliente, una disminución de los intermediarios, la importancia de la experiencia frente al destino y un mayor acento en la calidad y la sostenibilidad. Los grandes destinos turísticos españoles (entre los cuales, por supuesto, está la Comunidad Valenciana) llevan realizando importantes esfuerzos por adaptarse a estos cambios de tendencia a través de iniciativas novedosas (SegarraOña et al., 2012).

En cuanto a la pesca, el sistema actual está sumido en una profunda crisis no sólo económica sino también ecológica y social. La propia Unión Europea reconoce el fracaso de su Política Pesquera Común (Comisión Europea, 2011) y ha planteado una reforma que pretende dar un mayor protagonismo al propio sector de una forma organizada. Entre otras medidas, Bruselas propone incorporar las dimensiones biológica, económica y social de la pesca, un mayor papel para las instituciones regionales y un reforzamiento de las organizaciones de pescadores con el objetivo de que jueguen un papel decisivo a la hora de planificar las actividades pesqueras de sus miembros.

Con esta idea, el Fondo Europeo de Pesca (FEP), tal como establece el Reglamento (CE) 1198/2006, de 27 de julio de 2006, busca financiar una reestructuración del sector y se marca como uno de sus cinco ejes prioritarios el "desarrollo sostenible de las zonas de pesca", apoyando "medidas e iniciativas encaminadas a diversificar y potenciar el desarrollo económico en las zonas afectadas por la decadencia de las actividades de pesca". El turismo pesquero puede jugar un importante papel en este sentido, como vienen mostrando los trabajos que ya comienzan a analizar con cierta perspectiva este hecho (Chen, 2010; Cillari et al., 2012; Molina et al., 2010; Pardellas et al., 2011).

En todo caso, todavía son muchas las cuestiones a tener en cuenta y muchos interrogantes los que se plantean. Sin ánimo de abordar todos ellos, este trabajo, centrado en la Comunidad Valenciana, focalizará su atención en una cuestión básica en el planteamiento de estas iniciativas: la percepción -y, por tanto, la disposición- que los pescadores y sus instituciones, las Cofradías, tienen respecto a la pesca-turismo, las causas de confianza o desconfianza que les merece y, en definitiva, las oportunidades y amenazas que perciben en esta actividad. Cualquier intento de implantar una gestión eficaz y sostenible de pesca-turismo pasa por aprovechar el capital social existente en estas comunidades, integrando a los pescadores en nuevos modelos de co-gestión costera.

\section{LA PESCA-TURISMO EN ESPAÑA: PANORAMA GENERAL}

Muy recientemente, la pesca-turismo se alza como una iniciativa novedosa que puede alentar el desarrollo sostenible del litoral. Aún por desarrollar en la Comunidad Valenciana, en otros países europeos como Italia, Francia o Portugal llevan años promovién- 
dola con notable éxito y, dentro de España, en Galicia y Cataluña están trabajando en su impulso, creando una normativa propia y apostando por la puesta en práctica de iniciativas concretas (Padín y Aboy, 2010). También las Islas Canarias y las Baleares comienzan a legislar sobre esta materia.

No obstante, la pesca-turismo en nuestro país aún se encuentra en un estado embrionario; prueba de ello son las diferentes denominaciones con que la legislación se refiere a esta actividad: turismo pesquero, pesca-turismo, turismo marinero, ictioturismo... Nosotros entenderemos por pesca-turismo el "tipo de actividad de turismo pesquero o marinero desarrollada a bordo de embarcaciones pesqueras por parte de profesionales del sector, mediante contraprestación económica, que tiene por objeto la valorización y difusión de su trabajo en el medio marino, en la que los turistas embarcados no podrán ejercer la actividad pesquera" (Ley 33/2014 de 26 de diciembre por la que se modifica la Ley 3/2001, de 26 de marzo, de Pesca Marítima del Estado).

Con el apoyo financiero del FEP están apareciendo en España diferentes planes encaminados a crear, prácticamente desde la nada, un incipiente turismo pesquero (Molina et al., 2010). De hecho, el primer proyecto español de turismo pesquero fue en el año 2003 en la Cofradía de Lira, lo que contrasta con países como Italia que ya contaban con un reconocimiento legal de la pesca-turismo desde el año 1992. Otras iniciativas interesantes pero muy puntuales a lo largo de la costa española son, por ejemplo, las visitas guiadas al "Llanes Marinero" o visitas a la Lonja de Avilés, en Asturias; o "Águilas, El Mar y la Pesca”, en Murcia (Pérez y Espejo, 2012), que se ejecuta con financiación tripartita de la Administración General del Estado, la Comunidad Autónoma y el propio Ayuntamiento. También Andalucía ha participado en varias iniciativas transnacionales en el marco del Programa INTERREG III-B Mediterráneo Occidental con el apoyo del Fondo Europeo de Desarrollo Regional (FEDER). A partir de ello se han iniciado varias acciones de difusión de la cultura marinera mediante semanas culturales del mar compuestas por exposiciones fotográficas, proyecciones audiovisuales, conferencias, rutas gastronómicas, talleres divulgativos y educativos, etc. Otros ejemplos repartidos por toda la geografía serían las visitas temáticas guiadas de "Peñíscola Marinera" (Castellón) o la red de destinos marineros de nombre "Villas Marineras", que integra a 17 municipios de Galicia, Asturias y Cantabria, y trata de poner en valor y comercializar los recursos y productos de turismo marinero de estos destinos. En todo caso, estas iniciativas son puntuales y aisladas, y salvo en Cataluña y Galicia, aún no se han plasmado en proyectos concretos de pesca-turismo.

Uno de los proyectos más activos de los últimos años en materia de turismo y pesca fue el Proyecto SAGITAL ("Servicios de Adaptación para la Gestión de Iniciativas Turísticas Pesqueras en Áreas Litorales", 2005-2007), que se enmarcaba en la iniciativa EQUAL II del Fondo Social Europeo. Este proyecto se planteaba evaluar el grado de interés de las organizaciones de pescadores en temas de turismo pesquero, identificar los principales obstáculos jurídicos para su implantación y, por último, marcar diferentes estrategias a seguir. El estudio concluyó con la formulación de una propuesta de reglamentación para la regulación de las actividades de pesca-turismo y un estudio técnico sobre las condiciones de seguridad y de calidad de servicio que deben reunir los buques pesqueros para poder desarrollar estas actividades (Molina, 2013). 
Ciertamente, los proyectos españoles tropiezan con numerosos inconvenientes culturales, financieros y, sobre todo, legislativos (González-Pintado, 2012, Miret-Pastor et al., 2015). Sólo ahora, el Proyecto de Ley de Pesca Sostenible de 2014 reconoce explícitamente esta actividad, aunque todavía buena parte de las iniciativas siguen adoleciendo de la falta de un marco legal apropiado.

Además de la inseguridad jurídica (exceptuando Galicia, Cataluña y, recientemente, las Islas Baleares), los estudios, ciertamente escasos, sobre este tema destacan otro tipo de problemas para una implantación más generalizada de esta actividad, como la distribución irregular de las infraestructuras, la falta de seguimiento de los proyectos, las barreras que la regulación pueda imponer o la falta de experiencia y formación previa de los pescadores (Pascual, 2004, Pardellas et al., 2011, Molina, 2013). Son estas dos últimas trabas, regulación y formación, directamente relacionadas con las personas que, en última instancia, desarrollan la actividad, las que este trabajo indagará a partir de las propias percepciones de los pescadores.

\section{METODOLOGíA}

El cuerpo principal del material empírico de nuestro estudio está conformado por 32 entrevistas semiestructuradas a pescadores, todos ellos patrones de barco, y a patrones mayores y secretarios de cofradías de los puertos de Jávea, Denia, Gandía, Castellón, Cullera, Calpe, Sagunto y Vinaroz (Comunidad Valenciana). La interacción directa con estos informantes clave posibilitará clarificar, profundizar y analizar una información difícilmente accesible a partir de la escasa literatura existente.

Las entrevistas, de aproximadamente 45 minutos de duración, además de una serie de preguntas generales sobre información personal (edad, años ejerciendo como pescador, arte de pesca...), se centraron en la problemática actual en el mundo de la pesca de bajura, sus relaciones con las diferentes administraciones, así como sus conocimientos y visión (dificultades, condiciones, viabilidad...) de la pesca-turismo. El análisis de los discursos recogidos proporcionará la percepción social de los pescadores sobre las normativas impuestas y/o necesarias, así como los problemas específicos que se plantean. Se tratará también de captar la dimensión colectiva y simbólica de las opiniones y conductas individuales ante esta nueva actividad.

Todas las entrevistas, realizadas a lo largo del 2015, fueron grabadas y transcritas para, posteriormente, codificar los datos a partir de la herramienta informática Atlas.ti5, que facilitó la agrupación de citas, códigos y la creación de familias y redes. Los discursos recogidos se analizaron a partir de técnicas específicas de análisis de discurso para poner de manifiesto tanto la información directa como los presupuestos latentes y las estrategias discursivas empleadas (Taylor y Bodgan, 1994; Fairclough, 2013; Beltrán, 2016). Se pretende con ello proporcionar un marco de comprensión desde la propia perspectiva del grupo social que estamos investigando, así como ofrecer una detallada exposición de un escenario singular -la posible relación entre pesca costera y turismo- que nos permita profundizar y contribuir a los conocimientos sobre el tema objeto de estudio. Nuestra intención, de acuerdo con los objetivos planteados, es analizar un fenómeno en su propio entorno y, a partir de una metodología cualitativa, tratar de penetrar en los contextos de significación con los que 
el grupo opera, interpretando el sentido de la acción y su significado socio-cultural en un marco más amplio de cambios y conflictos socio-ambientales. Los extractos de discurso que mostraremos reflejan algunas de las ideas que aparecen repetidas una y otra vez en las entrevistas, así como aquellas otras que, aunque más excepcionales, podrían apuntar hacia ciertas tendencias aún no cristalizadas en la 'media' del colectivo de referencia.

\section{PESCA-TURISMO: OPORTUNIDADES Y RECELOS}

La pesca-turismo, como hemos ido viendo, se enfrenta a múltiples interrogantes a los que habría que dar respuesta antes de llevar a cabo un proyecto de este tipo. ¿Cómo hacer de la pesca-turismo un negocio rentable, a la vez que sostenible social y ambientalmente? Esta es una pregunta lícita y necesaria y, por ello, es importante abordar cuestiones como el producto a ofrecer, las tarifas, los costes de los seguros o de la seguridad, etc. Ahora bien, no podemos perder de vista una cuestión que está en la base de este proyecto: ¿tenemos los recursos humanos adecuados para tal empresa? Los escasos estudios que hay sobre el tema coinciden en resaltar la disposición de los pescadores para emprender tal tarea (Molina, 2013), aunque, como veremos, no dejan de manifestar también sus recelos. En particular, nos detendremos ante dos aspectos que pueden entorpecer su éxito: la posible escasa formación y capacitación por parte de quienes efectivamente tendrían que ofrecer el servicio y la posible dificultad de su aceptación y/o adecuación a una regulación de la actividad. En lo que sigue, analizaremos hasta qué punto ambos aspectos suponen un hándicap para el desarrollo de la pesca-turismo.

\subsection{La pesca-turismo como oportunidad}

A lo largo del siglo pasado, la actividad turística en la Comunitat Valenciana evolucionó desde el turismo urbano-cultural centrado en las grandes ciudades valencianas de la primera parte del siglo XX (Valencia, Alacant, Elx...) hacia el desarrollo de los destinos costeros vacacionales, dedicados al disfrute del buen clima al lado de la playa (Torrevieja, Benidorm, Gandía, Cullera, Benicassim, Peñíscola, etc.). Pequeñas ofertas de turismo de balnearios, turismo de "olas de mar", eventos y fiestas populares y poco más formaban parte de esa actividad novedosa que concentraba, en los escasos tiempos de descanso laboral y pocos recursos económicos de la mayoría de la población, el desplazamiento y estancia en otras localidades para el disfrute de dichos atractivos.

A partir de los años 60 el desarrollo económico general en Europa permitió aumentar los flujos turísticos. Ello repercutió en la Comunidad Valenciana en un aumento relativamente rápido de la demanda turística extranjera (Francia, Reino Unido, Alemania), a la que siguió la de los residentes en España (Madrid, Castilla-La Mancha, Castilla-León). El turismo en esta tierra creció en intensidad y diversidad, permitiendo la ganancia fácil de rentas y la inversión en nuevas infraestructuras y equipamientos turísticos. Numerosos espacios costeros se volcaron en la construcción de segundas residencias y otras ofertas de alojamiento, transformando la costa valenciana, antes salpicada por una flota pesquera artesanal de carácter familiar, en una suerte de interesada confusión entre turismo y construcción residencial (Cambrils, 2005). Ello provocó una modificación agresiva del paisaje 
natural en numerosos enclaves y el deterioro de la franja litoral, alterando bruscamente los ecosistemas marinos, y, por tanto, las estrategias de las unidades productivas de los pescadores litorales (Pascual y Santana, 2003), que aprovecharon la amplia oferta de trabajo generada por el turismo y la construcción para la obtención de nuevos ingresos. Así lo recuerdan algunos pescadores:

Trabajé unos años en la construcción, y ahí no te piden cursillos ni nada, tus ocho horas y pa casa. Pero no... (Arte menor, 29) ${ }^{2}$

Yo me tiré catorce años de cocina en Benidorm y bien. En los años cincuenta y nueve ya estaba yo yendo a Benidorm. En la mar pasé hambre, calamidades bastantes, siempre vas lleno de mierda porque te tiras al suelo a remendar y estás con los artes... de barro y demás ¿comprendes? Y allí no, en la cocina no me digas...chaqueta blanca, pantalón...claro, normal; sí, se nota la diferencia de una cosa con otra. (Arte menor, 12)

Yo estuve 4 años llevando un yate. En invierno lo mantenía y en verano te sacabas el seguro. De hecho hay quien lo hace aun. Claro, más experiencia que nosotros no hay. (PM, 5)

Estos informantes resaltan la dureza de la profesión, con ingresos inciertos y, además, una muy baja valoración social. Muestran también cómo el turismo, por su parte, ejercía cierto atractivo y, junto con la construcción, aportaba una alternativa segura, fácil y bien remunerada. Eso apreciaron muchos pescadores que, durante unos años, cambiaron las redes por cocinas y ladrillos. "Pero, no...", se queja el pescador anterior: por mucho que hubieran ensayado otras profesiones, "ser pescador se lleva en la sangre" -como una y otra vez se repite en las entrevistas- y prácticamente todos ellos volvieron a la pesca. Hoy no se parte, por tanto, desde cero; los pescadores han estado en contacto directo con el turismo. Bien por la coincidencia espacial, bien por cuestiones laborales, el del turismo es un mundo que no les resulta ajeno.

Disponen, además, de unos conocimientos imprescindibles difíciles de alcanzar de otra manera: a ser pescador "no enseña nadie", "nos enseñaron la práctica y tenemos la práctica". No serían, por tanto, sus conocimientos, o más concretamente, la falta de ellos, una traba para implantar la pesca-turismo. Ciertamente, las comunidades locales, en cuanto tales, son quienes mejor conocen su espacio circundante y ejercen así un 'control' del conocimiento sobre el territorio que está basado en su experiencia, su concreta ubicación territorial y su memoria colectiva. Estos conocimientos entrañan la identificación de un espacio en el que faenar (rutas bénticas y pelágicas, lugares identificados por nombres específicos, puntos de referencia, recursos, estación y tasas de explotación...); las diferentes especies de peces, su comportamiento y su entorno; la meteorología, que se relaciona con la navegación y el calendario de pesca, etc. (Féral, 2004). Los pescadores han acumulado un importante repertorio de saberes que les ha permitido ejercer cierto control de un medio complejo y siempre

2 En adelante, la mención a cada informante hace referencia a su posición social, en el caso de patrones mayores (PM, 1; PM, 2...) o secretarios de cofradías (S, 1; S, 2...). Las citas de los pescadores se identifican por el arte que suelen practicar. Los resaltados en negrita en las citas de los informantes son nuestros. En las citas de los informantes que, entrecomilladas, se incorporan al texto, omitimos su identificación precisa para no recargarlo. 
cambiante adecuando su comportamiento (social) al comportamiento natural. Han 'vivido' cómo los peces se reproducen, se ocultan, buscan alimento... y, por tanto, su conocimiento va más allá del mero dominio de unas técnicas, extendiéndose a interpretar y descifrar lo que sucede en 'su' medio natural (Martínez-Novo, 2016).

Aunque, ciertamente, carecen de un procedimiento metodológico consensuado para obtener ese conocimiento, cada pescador y cada grupo emplea sus propias habilidades intelectuales, sensitivas y experienciales para decidir cómo actuar en cada momento, lugar y situación (Florido, 2002):

Al mar se aprende yendo y trabajando. Y punto. [Yendo] a una academia nunca aprenderás. No hay curso que sirva para algo. (PM, 19)

La experiencia así acumulada, que no es más que su vida día a día, conformaría la base esencial sobre la que se construye el conocimiento en el que se cimenta la pesca-turismo:

Hombre, esto siempre se ha sabido. Se sabe. Siempre hay más pescado cuando más cerca estás de las montañas de dentro del mar. Entonces, hay sitios donde se ha pescado siempre, los abuelos han transmitido estos... nosotros decimos "senyes", hay señas, en castellano no sé cómo se llama. Por ejemplo, desde dentro del mar, esto es una montaña y veo esta anza, entonces la giro por el otro canto de montaña y yo estoy dentro del mar y enfilo y digo por aquí y he cogido mucho pescado. (Arte menor, 17)

Yo he ido con mi padre y mi padre me ha dicho: vamos a calar ahí, al "llit del Alguer”. Y bueno, vale. Y hemos ido y dices: ¿y por qué mañana no vamos? Y dice: porque ya es "fosca", porque la luna ya ha pasado, la luna fuerte ya ha pasado, ya viene la "foscor". O sea viene la luna baja y entonces ya no quieren ir allí, quieren ir en ese tiempo determinado, entonces parece ser que sí, que se coge pescado cuando la luna está con las condiciones que toca. (Arte menor, 18)

Este conocimiento colectivo ("se sabe"), acumulado, contrastado, reformulado y transmitido de generación en generación ("mi padre me ha dicho") y adaptado a las condiciones medioambientales locales ("hay señas", "la luna ya ha pasado") es imposible fuera de las comunidades de pescadores: "en una academia nunca aprenderás". Por eso, para ellos pescar es mucho más que un trabajo, es un oficio o profesión, la cual solo es posible al estar inserta en todo un modo de vida. En consecuencia, para los pescadores, la pesca-turismo va más allá que unos meros ingresos complementarios; es también una oportunidad importante de poner en valor su forma de vida y sus conocimientos:

También nos podía ayudar un poquito en el sector para los días mal. Hay días que sales a pescar y sólo traes algas... Hay días que echas la red y preferirías quedarte en tu casa... Dos días que llevas a gente, echas un poquito de red, unos anzuelos, ven cómo trabajas, cómo... valorarían más... tú tendrías un poquito de recurso económico para ayudar. Pienso, eh, a lo mejor me engaño. El turismo lo agradecería también, creo. (Arte menor, 17) 
Y no es el dinero, es el promocionar nuestra vida y nuestro oficio. El proyecto de pesca-turismo no es para ganarnos la vida, ni en broma. Es para dar a conocer nuestro patrimonio, nuestra vida, nuestra gastronomía, promocionar nuestro producto. Nosotros, por razón de nuestro trabajo, somos los grandes desconocidos. Nos vamos de madrugada, venimos a la tarde de trabajar y la gente no valora lo que es el pescado. $(\mathrm{PM}, 3)$

El interés turístico por su forma de vida también lo deduce el pescador de su propia experiencia. Un pescador también es un turista que, en su tiempo libre, ha sentido curiosidad por otras formas de vida:

Yo creo que sería súper bueno. Yo voy al interior y me gusta conocer su cultura, cómo viven y de qué viven (si es de la viña, de las patatas, del espárrago como en Navarra...). Lo mismo sería para los turistas: qué pescado y cómo se captura, cómo se cocina... Es interesante. $(\mathrm{S}, 1)$

Sabedores del escaso conocimiento de su profesión por el resto de la sociedad e, incluso, de la desvalorización social de que son objeto, muchos pescadores entrevistados ven en la pesca-turismo una forma de dar a conocer y poner en valor la pesca, su cultura y su producto.

No obstante, desde instancias administrativas y empresariales suele aducirse que los pescadores tradicionales carecen del espíritu innovador necesario para emprender iniciativas como la pesca-turismo. Los pescadores son conscientes de esos tópicos tan extendidos, como que 'sólo saben pescar', o que sus principios, considerados arcaicos y obsoletos, cuestionan su capacidad de llevar a cabo iniciativas emprendedoras (García Allut, 2010). Sin embargo, en esas supuestas carencias y defectos, ellos cifran precisamente su valor. Argumentan que no solo son ellos quienes, desde sus cofradías, han tenido la iniciativa de proponer la actividad de pesca-turismo, sino que se ven a sí mismos como constantes innovadores, debido a la necesidad permanente de adaptarse a las siempre cambiantes condiciones del mundo marino (Féral, 2004). Pero los continuos inventos a que ello les obliga no se perciben bajo la categoría abstracta y tecno-burocrática de la innovación (Martínez-Novo et al., 2016), así como tampoco construyen su identidad bajo la categoría neoliberal de emprendedores, pese a percibir su actividad como una en la que emprenden sin cesar. El pescador tradicional, aunque a menudo es su propio empresario y aunque habitualmente improvisa soluciones nuevas, no construye su identidad en ninguno de esos términos, sino en el único que para ellos tiene sentido: ser pescador: "yo soy pescador, eso es ya empresario" (Arte menor, 15).

\subsection{Recelos ante la implantación de la pesca-turismo}

En el caso español, la gestión de las pesquerías costeras se basa en un modelo mixto donde la acción centralizada del Estado (Ministerio y Comunidades Autónomas) se articula con la auto-organización de los pescadores en el seno de las Cofradías (Franquesa, 2004; Lizcano et al., 2016). Estas actúan como órganos de consulta y colaboración con las administraciones competentes y poseen la "capacidad de obrar para el cumplimiento 
de sus fines" (Ley 3/2001, de 26 de marzo de Pesca Marítima del Estado). Pese a lo cual, su papel en los procesos de elaboración y toma de decisiones les resulta cada vez más complicado y ajeno (Herrera et al., 2015):

A veces sueltan una ley y dicen: consultado con el sector. ¡Consultado... de aquella manera! Te mandan un papelito y al día siguiente te sacan la orden y eso es lo que no puede ser. $(S, 9)$

Y ello se traduce en un problema de pérdida de legitimidad de las instituciones políticas por parte de los pescadores, problema que se hace más profundo a medida que se distancia el centro de decisión:

Si no saben ni para qué ponen las leyes... Ahora, por ejemplo, están con el tema de la contaminación, pegando palos de ciego. Que me parece muy bien que la erradiquen totalmente, pero, por ejemplo, el tema que para mear... en la tellina, nos obligan a salir a tres millas de la costa para mear, ;pero si todo el mundo sabe que lo primero que hace la gente al entrar a la playa es mear! (Arrastre, 22)

Esta ignorancia por parte de los centros de decisión el pescador la cifra en su progresivo alejamiento de las situaciones reales y concretas, lo que impide a las medidas políticas conocer las particularidades de cada caso:

Y lo que pasa en la comunidad europea es que es como si todos los mares fueran iguales. A ellos les da igual que estés en el Cantábrico que en el Atlántico y no es lo mismo. Y ellos se cogen a la normativa de un tío que se sienta en unos despachos y dice eso tiene que ser así y punto. (PM, 25)

La lejanía y falta de transparencia de las decisiones, la incomprensión de las normas o la homogeneización de las regulaciones (Lucas y Kirit, 2009, Herrera et al. 2015) abren una brecha de confianza entre administración y administrados que se hace cada vez más difícil de cerrar. Una brecha que se acentúa ante una nueva alternativa, como la pescaturismo, que exigiría una nueva regulación que suponen sería fuente de nuevos problemas:

Pero lo que no se comprende es que exijan tanta seguridad; que hay que tenerla y la tenemos. Lo que no puede ser es que para ir en una embarcación te pidan una seguridad del $300 \%$ de la gente que lleves en la barca. Además, el seguro que tenemos que pagar es muy elevado. Si, además del seguro elevado, pones toda la seguridad que te exigen habrá más material de salvamento que gente embarcada. (Arte menor, 25)

El sinsentido, contradicción o paradoja con que concluye este pescador su argumento es habitual en otras entrevistas:

Igual que lo de la pesca-turismo... pero es lo de siempre, que tropezamos con la administración. Perdona, las administraciones, que hay varias. Es necesaria, sí, 
y una quimera. Necesaria para que el sector no desaparezca, pero una quimera porque ya se encarga la Administración de que no se haga. Por cuestión de competencias entre el Ministerio de Pesca, el Ministerio de Fomento, la Consellería de Pesca... porque aquí son 25 administraciones y cada una va por libre. Lo ordena Fomento, y es un Ministerio de Madrid y en Madrid que yo sepa, agua no hay, mar no la hemos visto. $(S, 6)$

La Administración es necesaria para poner orden, pero su multiplicación genera un caos; se quiere ordenar el mar con criterios de tierra (Madrid); o la administración simula interés por promocionar el conocimiento de una actividad (la pesca artesanal) que ella misma ha estado erosionando:

Eso [la pesca-turismo] es una camama, es un caramelo envenenado. Quieren cubrir alguna parte de aquel mercado. Pero claro, es decir, pues vamos a generar la necesidad de... joiga si por un lado usted está lidipendiando [vilipendiando] a los pescadores, ¿ahora quiere decir: salga usted con estos pescadores?! (S, 13)

Esta desafección hacia los poderes públicos llega a interpretarse, en ocasiones, como una decidida amenaza a su supervivencia, incluso como una voluntad soterrada de hacerles desaparecer como comunidad: "Leyes. Pues ¿qué quieren? Para mí, hacértelo difícil, como para decir: acúrrete ya y retírate, que molestas".

Y, a pesar de ello, los pescadores reconocen -como hacía S, 6- que necesitan de la administración para proteger sus intereses, que su control es necesario. De ahí las paradojas o contradicciones que perciben de continuo: esa misma administración de la que tanto desconfían es la que podría, en principio, garantizar el éxito de la nueva actividad de pesca-turismo. Pero sólo en principio: la pesca-turismo sólo llegará a buen puerto si administraciones públicas y cofradías trabajan conjuntamente. De una forma u otra, la idea de co-gestión, que fundamenta la estrategia de GIZM, queda reflejada en todas las entrevistas:

Sí, poner [la Consellería] unas normas de seguridad... Pero... las cofradías tienen que ejercer un papel; $\sin (. .$.$) sin cofradías esto será la selva, un desastre. Si no$ lo gestionara la cofradía sería un desastre, porque cada uno iría por su senda. (Arte menor, 2)

Lo que pedíamos era una uniformidad conforme estaba hasta la fecha, para tener una seguridad para, para poder trabajar. Y hemos quedado en este momento, ahora en verano, en reunirnos con todos los ayuntamientos de la provincia de Valencia y con la patronal de turismo y... el Ministerio de Fomento y a ver si se puede... si se aclara. $(\mathrm{S}, 5)$

A pesar del tiempo transcurrido, una vez más las dificultades ya detectadas en los proyectos de demostración para la implantación del GIZC parecen no haber encontrado solución: la participación de los interesados sigue sin ser adecuada (a menudo es, incluso, inexistente) y los sistemas burocráticos rígidos y la falta de coordinación entre las instancias administrativas competentes no sólo entorpecen nuevas alternativas, sino que también limitan la creatividad y adaptabilidad local. (Comisión Europea, 2000). 


\section{CONCLUSIONES}

Tanto este como otros trabajos previos indican que el pescador tradicional no rechaza la implantación del turismo pesquero. De hecho, las encuestas llevadas a cabo por el Proyecto Sagital $^{3}$ indicaron unos niveles de aceptación hacia esta actividad por parte de las cofradías que iban desde el 91\% en 2007 al 100\% de aceptación en 2012.

Estos datos cuantitativos son confirmados por nuestro análisis cualitativo. El pescador valenciano ha convivido con el turismo desde hace muchos años, ha compartido territorio y puertos, se han producido determinadas relaciones de conflicto (con la pesca deportiva) o de colaboración (como patrones de barcas de recreo). Incluso entre el turismo y la pesca ha habido un continuo flujo de trabajadores. Por tanto, el turismo no es un mundo ajeno para el pescador.

La pesca-turismo es generalmente aceptada por los pescadores y no sólo por la posibilidad de que aporte ingresos adicionales, sino sobre todo por la posibilidad que supone para dar a conocer y poner en valor su cultura, su trabajo y su producto. El pescador expresa continuamente la sensación de ser una comunidad aparte y accede de buen grado a que otros (el turista, la gente de tierra) pueda embarcarse, conocer y valorar su actividad y su producto. Con ello, no sólo trata de romper los tópicos en torno a él, también espera que acabe reflejándose en la demanda y precio del pescado.

Pero la aceptación de esta actividad contrasta con la falta de legitimidad que mantienen hacia aquellos que habrían de implementarla: la administración. Los pescadores no pierden oportunidad de mostrar la desconfianza que les genera la ley y la administración. Para ellos es una fuente de problemas y papeleo. Se quejan de la burocracia y de la lejanía de unas leyes dictadas desde muy arriba y que nada o poco tienen que ver con su realidad, lo que temen acabe dándose nuevamente en la legislación sobre pesca-turismo. No obstante, la actitud de nuestro pescador hacia la administración que debe reglamentar esta práctica es ambigua, si no paradójica: por un lado, para él es una fuente de problemas, despropósitos y arbitrariedad, pero, por otro, reconoce sin paliativos la necesidad de su intervención y de encontrar el modo de colaborar con ella.

Cabe concluir, por tanto, que cualquier intento de implantar una gestión eficaz y sostenible de la pesca-turismo pasa por aprovechar el capital social existente en estas comunidades, integrando a los pescadores en un nuevo modelo de co-gestión. Como la pesca-turismo estará protagonizada necesariamente por los pescadores, deben ser ellos (y sus instituciones, las Cofradías) los protagonistas de su diseño e implantación. Estas conclusiones sobre pesca-turismo están muy en la línea de lo que debe ser en general la gestión pesquera y por lo que apuesta la nueva normativa europea. En todo caso, si de verdad hay un cambio de enfoque en la política pesquera, el diseño de la necesaria normativa y la implantación de la pesca-turismo puede ser una gran oportunidad para demostrarlo.

Este trabajo se ha centrado en analizar la visión de los pescadores sobre la pescaturismo. Aunque el estudio está acotado en una región muy concreta, creemos que sus reflexiones y conclusiones pueden ser extensibles a otras muchas partes del mundo, sobre

3 Véase Proyecto Sagital, http://www.fundame.org/portfolio-item/proyecto-sagital-servicios-de-adaptacion-para-la-gestion-de-iniciativas-turistico-pesqueras-en-areas-litorales/ 
todo en el momento de diseñar y adaptar, a partir del capital social disponible (conocimientos y prácticas de los pescadores locales o sus instituciones propias), regulaciones de futuras experiencias.

\section{BIBLIOGRAFÍA}

AGARWAL, S. (2002): "Restructuring seaside tourism. The resort lifecycle", Annals of Tourism Research, 29, pp. 5-55.

BELTRÁN, M. (2016): Dramaturgia y hermenéutica: para entender la realidad social, Madrid, Centro de Investigaciones Sociológicas.

BUTLER, R. (1980): “The concept of the tourist area life-cycle of evolution: implications for management of resources", Canadian Geographer, 24 (1), pp. 5-12.

CAMBRILS, J.C. (2005): "Reposicionamiento en destinos maduros: El caso de Gandía", Estudios turísticos, 166, pp. 115-131.

CHEN, C-L. (2010): "Diversifying fisheries into tourism in Taiwan: Experiences and prospects”, Ocean \& Coastal Management, 53 (8), pp. 487-492.

CILLARI, T. et al. (2012): "The use of bottom longline on soft bottoms: An opportunity of development for fishing tourism along a coastal area of the Strait of Sicily (Mediterranean Sea)", Ocean \& coastal management, 55, pp. 20-26.

COMISIÓN EUROPEA (2011): "Reform of the Common Fisheries Policy", Brussels, disponible en http://eur-lex.europa.eu/legal-content/EN/TXT/PDF/?uri=CELEX:5201 1DC0417\&from=EN [12 de junio de 2015].

COMISIÓN EUROPEA (2007): Comunicación de la Comisión al Parlamento Europeo, al Consejo, al Comité Económico y Social Europeo y al Comité de las Regiones. Una política marítima integrada para la Unión Europea, COM(2007) 575 final, Bruselas.

COMISIÓN EUROPEA (2000): Comunicación de la Comisión al Consejo y al Parlamento Europeo. Sobre la Gestión Integrada de las Zonas Costeras: una estrategia para Europa (COM(2000) 547 final), Bruselas.

FAIRCLOUGH, N. (2013): Critical discourse analysis: the critical study of language, New York: Routledge.

FAIRWEATHER, J., MASLIN, C. y SIMMONS, D.G. (2005): "Environmental values and response to ecolabels among international visitors to New Zealand", Journal of Sustainable Tourism, 13 (1), pp. 82-98.

FARRELL, B.H. (2008): "Cooperative tourism and the coastal zone", Coastal Management, 14, Issue 1-2, pp. 113-130.

FÉRAL F. (2004): "Maritime societies, fisheries law and institutions in the Western Mediterranean". Fisheries Technical Paper 420. Roma, FAO.

FLORIDO, D. (2002): “Los sentidos y el 'saber hacer' de los pescadores andaluces", Demófilo. Revista de Cultura Tradicional de Andalucía, 1, pp. 139-156.

FRANQUESA, R. (2004): "Fishermen guilds in Spain (Cofradias): economic role and structural changes" IIFET 2004 Japan Proceedings, 14, disponible en https:// www.researchgate.net/publication/265991036_FISHERMEN_GUILDS_IN_SPAIN_ COFRADIAS_ECONOMIC_ROLE_AND_STRUCTURAL_CHANGES [6 de marzo de 2014]. 
GARCÍA-ALLUT, A. (2010): "Sostenibilidad ambiental, desarrollo territorial y RSE", Revista Ambienta, $\mathrm{n}^{\circ} 93$ disponible en http://www.revistaambienta.es/WebAmbienta/ marm/Dinamicas/pdfs/versionpdf/Allut.pdf) [15 de abril de 2015].

GONZALEZ-PINTADO, J.F. (2012): Perspectiva jurídica de la gestión y conservación de los recursos pesqueros en España. La pesca-turismo como opción de futuro. Tesis doctoral, disponible en http://oa.upm.es/14689/1/JOSE_FERNANDO_GONZALEZ_ PINTADO.pdf [18 de enero de 2016]

HERRERA-RACIONERO, P., LIZCANO, E. y MIRET-PASTOR, L. (2015): “'Us' and 'them'. Fishermen from Gandía and the loss of institutional legitimacy". Marine Policy, 54, pp. 130-136.

IOANNIDES, D. y KEITH D. (1998): "Neo-fordism and flexible specialization in the travel industry", en The economic geography of the tourist industry: a supply-side analysis" (Ioannides, D. y Debagge, K., eds.), London (UK): Routledge, pp. 99-122.

JOB, H. y PAESLER, F. (2013): "Links between nature-based tourism, protected areas, poverty alleviation and crises-The example of Wasini Island (Kenya)" Journal of Outdoor Recreation and Tourism, 1 (2), pp. 18-28.

KNOWLES, T. y CURTIS, S. (1999): “The Market Viability of European Mass Tourist Destinations. A Post-Stagnation Life-cycle Analysis", International Journal of Tourism Research, 1 (4), pp. 87-96.

LIZCANO, E., HERRERA-RACIONERO, P., MASCARELL, Y. y VALLEJOS, A. (2016): "The Spanish Mediterranean coast guilds: their role in the Integrated Coastal Zone Management”, Comunicación en MSEAS 2016, 30 May - 3 June 2016, Brest, France.

LUCAS, E.Y. y KIRIT, R. (2009): Fisheries-Marine Protected Area-Tourism Interactions in Moalboal, Cebu, Philippines. Coastal Management, 37 (5), pp. 480-490.

MARTÍNEZ-NOVO, R., LIZCANO, E., HERRERA-RACIONERO, P. y MIRET-PASTOR, L. (2016): "Innovation or 'Inventions'? The conflict between latent assumptions in marine aquaculture and local fishery" Public Understanding of Science, doi: $10.1177 / 0963662516651358$.

MIRET-PASTOR, L., MUÑOZ ZAMORA, C., HERRERA-RACIONERO, C. y MARTÍNEZ NOVO, R. (2015): “Análisis regional del turismo pesquero en España”, Revista de Análisis Turístico, 20, pp. 23-28.

MOLINA, A. (2013): Diagnóstico estratégico de la actividad de Pesca Turismo en España, Madrid, Ministerio de Agricultura, Alimentación y Medio Ambiente.

MOLINA, A., GONZÁLEZ, J.F. y GARCÍA, C. (2010): "El turismo pesquero como instrumento de apoyo al desarrollo sostenible en zonas litorales: la experiencia del proyecto Sagital", XIV International Congress on Project Engineering disponible en http://oa.upm.es/8159/1/INVE_MEM_2010_81564.pdf [3 de marzo de 2016]

ÖNDER, Ö., AYKAN, O. y KUMRAL, N. (2009): “An Empirical Analysis of the Determinants of International Tourism Demand: The Case of Izmir", European Planning Studies, 17 (10), pp. 1525-1533.

PADÍN, C. y ABOY, S. (2010): “Nuevos productos turísticos: la apuesta por el turismo marinero en Galicia”, Revista de Estudios Politécnicos, 7 (14), pp. 55-83. 
PAPATHEODOROU, A.; ROSSELLÓ, J. y XIAO, H. (2010): "Global Economic Crisis and Tourism: Consequences and Perspectives", Journal of Travel Research, 49 (1), 39-45.

PARDELLAS, X.X., PADÍN, C. y ABOY, S. (2011): “Turismo pesquero: experiencias en Europa y España”, Papeles de Economía Española, 128, pp. 221-227.

PASCUAL, J. (2004): "Notas de investigación. Las investigaciones sobre la pesca en Canarias: entre las reservas marinas y las nuevas formas de pesca turismo", Pasos, 2 (2), pp. 295-306.

PASCUAL, J. y SANTANA, A. (2003): "Pesca y turismo: conflictos, sinergias y usos múltiples en Canarias", PH: Boletín del Instituto Andaluz del Patrimonio Histórico, 11(44), pp. 86-97.

PÉREZ, P. y ESPEJO, C. (2012): La pesca como factor de desarrollo del turismo sostenible. El caso de águilas (Murcia), Cuadernos de Turismo, 30, pp. 267-284.

SEGARRA-OÑA, M.V. et al. (2012): "The effects of localization on economic performance: Analysis of Spanish tourism clusters", European Planning Studies 20 (8), pp. 1319-34.

TAYLOR, S. y BOGDAN, R. (1984): Introduction to qualitative research methods: The search for meaning, New York, John Wiley and Sons.

TORRES, R. (2002): “Cancun's tourism development from a Fordist spectrum of analysis", Tourist Studies, 2, pp. 87-116. 
